\title{
Updated CDC Recommendations for Using Artemether-Lumefantrine for the Treatment of Uncomplicated Malaria in Pregnant Women in the United States
}

\author{
Sarah-Blythe Ballard, MD, PhD ${ }^{1,2}$; Allison Salinger, $\mathrm{MPHc}^{2,3}$; Paul M. Arguin, MD²; Meghna Desai, $\mathrm{PhD}^{2}$; Kathrine R. Tan, MD²
}

Malaria infection during pregnancy is associated with an increased risk for maternal and fetal complications. In the United States, treatment options for uncomplicated, chloroquine-resistant Plasmodium falciparum and P. vivax malaria in pregnant women are limited to mefloquine or quinine plus clindamycin (1). However, limited availability of quinine and increasing resistance to mefloquine restrict these options. Strong evidence now demonstrates that artemetherlumefantrine (AL) (Coartem) is effective and safe in the treatment of malaria in pregnancy. The World Health Organization (WHO) has endorsed artemisinin-based combination therapies (ACTs), such as AL, for treatment of uncomplicated malaria during the second and third trimesters of pregnancy and is currently considering whether to add ACTs, including AL, as an option for malaria treatment during the first trimester $(2,3)$. This policy note reviews the evidence and updates CDC recommendations to include $\mathrm{AL}$ as a treatment option for uncomplicated malaria during the second and third trimesters of pregnancy and during the first trimester of pregnancy when other treatment options are unavailable. These updated recommendations reflect current evidence and are consistent with WHO treatment guidelines.

\section{Background}

Each year, approximately 1,700 cases of imported malaria occur in the United States; approximately $630(37 \%)$ of these cases occur in women, including 5\%-6\% who are pregnant at the time they are infected (4). Treatment options for uncomplicated, chloroquine-resistant $P$. falciparum and $P$. vivax malaria infections in pregnant women in the United States are threatened by the spread of mefloquine resistance in Southeast Asia. Having only one quinine and mefloquine manufacturer in the United States can adversely affect access. In 2009, the Food and Drug Administration (FDA) approved AL for the treatment of uncomplicated malaria. At that time, this combination was not approved for use in pregnancy because animal research data indicated a potential association with poor pregnancy outcomes, and insufficient human data were available. Since then, global experience has contributed substantial evidence of the safety and efficacy of AL throughout pregnancy. Given the need for an additional option to treat uncomplicated malaria in pregnant women in the United States, a systematic review of the literature was performed to evaluate the safety and efficacy of AL use during pregnancy, and findings were used to update CDC recommendations.

\section{Methods}

A systematic review of English-language research articles listed in PubMed was conducted using the keywords "artemether," "lumefantrine," "Coartem," and "malaria in pregnancy." Clinical trials, observational studies, meta-analyses, and case reports of uncomplicated malaria treatment during pregnancy were included. Studies that did not include treatment or pregnancy outcomes were excluded, as were studies that did not identify the trimester of treatment. Review article and metaanalysis references were examined for additional primary source articles for inclusion. Online search results were compiled and deduplicated. Two independent reviewers determined the relevance of each article to the research objective based first on title, then abstract, then full text. If reviewers had discordant findings from title or abstract review, the article was included in the next review phase. The following data were abstracted and reviewed: participant age; geographic location; parity; reason for drug treatment (uncomplicated versus severe malaria); trimesters during which treatment occurred; medication dose administered; treatment duration; treatment outcomes; and pregnancy outcomes, which included miscarriage (pregnancy loss at $<28$ weeks' gestation), stillbirth (pregnancy loss at $\geq 28$ weeks' gestation), preterm birth ( $<37$ weeks' gestation), low birth weight $(<2,500 \mathrm{~g})$, congenital abnormalities, and any maternal adverse events reported.

\section{Rationale and Evidence}

Systematic review results. In the initial search, 1,726 articles were identified. After excluding four articles during deduplication, 1,534 during title review, 94 during abstract review, and 73 after full text review, 21 articles remained and were included in the review.

Efficacy. One meta-analysis (5) and five randomized openlabel controlled trials performed in Uganda and Thailand examined the efficacy of ACTs for uncomplicated $P$. falciparum in women during their second and third trimesters of pregnancy and found cure rates $\geq 94.9 \%$, with ACTs performing equal to or better than quinine-based regimens (Table 1) $(6-10)$. A meta-analysis of African and Asian studies found lower but statistically similar treatment failure rates by days $28-63$ in 
Morbidity and Mortality Weekly Report

TABLE 1. Findings of randomized trials of artemisinin-based regimens for treatment of malaria in pregnancy

\begin{tabular}{|c|c|c|c|c|c|c|}
\hline $\begin{array}{l}\text { Author, } \\
\text { publication year }\end{array}$ & Country & $\begin{array}{l}\text { Indication for } \\
\text { treatment }\end{array}$ & Drug regimen & $\begin{array}{c}\text { No. of } \\
\text { participants }\end{array}$ & $\begin{array}{l}\text { Follow-up } \\
\text { time (days) }\end{array}$ & $\begin{array}{c}\text { Treatment outcome, } \\
\%(95 \% \mathrm{Cl})\end{array}$ \\
\hline $\begin{array}{l}\text { McGready, et al., } \\
2000^{*}\end{array}$ & Thailand & $\begin{array}{l}\text { Uncomplicated } \\
\text { P. falciparum, second } \\
\text { and third trimesters }\end{array}$ & $\begin{array}{l}\text { 1. MQ } 25 \mathrm{mg} / \mathrm{kg} \times 1 \text { and As } 4 \mathrm{mg} / \mathrm{kg} / \mathrm{d} \times 3 \mathrm{~d} \\
\text { 2. Q } 10 \mathrm{mg} / \mathrm{kg} \text { q8hr } \times 7 \mathrm{~d}\end{array}$ & $\begin{array}{l}66 \\
42\end{array}$ & $\begin{array}{l}63 \\
63\end{array}$ & $\begin{array}{l}\text { Cure } 98.2(94.7-100)^{\dagger} \\
\text { Cure } 67.0(43.3-90.8)^{\dagger}\end{array}$ \\
\hline $\begin{array}{l}\text { McGready, et al., } \\
2001^{\S}\end{array}$ & Thailand & $\begin{array}{l}\text { Uncomplicated } \\
\text { P. falciparum, second } \\
\text { and third trimesters }\end{array}$ & $\begin{array}{l}\text { 1. As } 2 \mathrm{mg} / \mathrm{kg} / \mathrm{d} \times 7 \mathrm{~d} \\
\text { 2. Q } 10 \mathrm{mg} / \mathrm{kg} \mathrm{q} 8 \mathrm{hr} \times 7 \mathrm{~d} \text { and } C L 5 \mathrm{mg} / \mathrm{kg} \\
\text { q8hr } \times 7 \mathrm{~d}\end{array}$ & $\begin{array}{l}64 \\
65\end{array}$ & $\begin{array}{l}42 \\
42\end{array}$ & $\begin{array}{l}\text { Cure } 100 \\
\text { Cure } 100\end{array}$ \\
\hline $\begin{array}{l}\text { McGready, et al., } \\
2005^{\oplus}\end{array}$ & Thailand & $\begin{array}{l}\text { Uncomplicated } \\
\text { P. falciparum, second } \\
\text { and third trimesters }\end{array}$ & $\begin{array}{l}\text { As } 4 \mathrm{mg} / \mathrm{kg} / \mathrm{d} \times 3 \mathrm{~d} \text { and } \mathrm{A} 20 \mathrm{mg} / \mathrm{kg} / \mathrm{d} \times 3 \mathrm{~d} \\
\text { and P } 8 \mathrm{mg} / \mathrm{kg} / \mathrm{d} \times 3 \mathrm{~d} \\
\text { Q } 10 \mathrm{mg} / \mathrm{kg} \mathrm{q} 8 \mathrm{hr} \times 7 \mathrm{~d}\end{array}$ & $\begin{array}{l}39 \\
42\end{array}$ & $\begin{array}{l}63 \\
63\end{array}$ & $\begin{array}{l}\text { Cure } 94.9(81.37-99.11)^{\dagger, * *} \\
\text { Cure } 63.4(46.9-77.4)^{\dagger, t \dagger}\end{array}$ \\
\hline 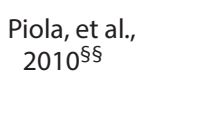 & Uganda & $\begin{array}{l}\text { Uncomplicated } \\
\text { P. falciparum }\end{array}$ & $\begin{array}{l}\text { 1. AL } 20 / 120 \mathrm{mg} 4 \text { tabs at } 0 \text { and } 8 \mathrm{hr} \times 1 \mathrm{~d} \text {, } \\
\text { then BID } \times 2 \mathrm{~d} \\
\text { 2. Q } 10 \mathrm{mg} / \mathrm{kg} \mathrm{q} 8 \mathrm{hr} \times 7 \mathrm{~d}\end{array}$ & $\begin{array}{l}152 \\
152\end{array}$ & $\begin{array}{l}42 \\
42\end{array}$ & $\begin{array}{l}\text { Cure } 99.3(96.0-99.9)^{\dagger, 9 \uparrow} \\
\text { Cure } 97.6(93.1-99.5)^{\dagger, * * *}\end{array}$ \\
\hline $\begin{array}{l}\text { Kaye, et al., } \\
2008^{++\dagger}\end{array}$ & Uganda & $\begin{array}{l}\text { Uncomplicated } \\
\text { P. falciparum, second } \\
\text { and third trimesters }\end{array}$ & $\begin{array}{l}\text { 1. AL } 20 / 120 \mathrm{mg} 4 \text { tabs at } 0 \text { and } 8 \mathrm{hr} \times 1 \mathrm{~d} \text {, } \\
\text { then BID } \times 2 \mathrm{~d} \\
\text { 2. LapDap } \times 3 \mathrm{~d}\end{array}$ & $\begin{array}{l}57 \\
57\end{array}$ & $\begin{array}{l}28 \\
28\end{array}$ & $\begin{array}{l}\text { Cure } 100 \\
\text { Cure } 100\end{array}$ \\
\hline
\end{tabular}

Abbreviations: $\mathrm{A}=$ atovaquone; $\mathrm{AL}=$ artemether-lumefantrine; $\mathrm{AQ}=$ amodiaquine; $\mathrm{As}=$ artesunate; $\mathrm{BID}=\mathrm{twice}$ daily; $\mathrm{Cl}=\mathrm{confidence} \mathrm{interval;} \mathrm{d}=$ days; $\mathrm{hr}=$ hour $(\mathrm{s})$; $\mathrm{kg}=$ kilogram; LapDap = chlorproguanil-dapsone; $\mathrm{mg}=$ milligram; $\mathrm{MQ}=$ mefloquine; $\mathrm{P}=$ proguanil; $\mathrm{PCR}=$ polymerase chain reaction; $\mathrm{Q}=\mathrm{quinine} ; \mathrm{qd}=$ once daily; $\mathrm{q} 8 \mathrm{hr}=$ every 8 hours.

* McGready R, Brockman A, Cho T, et al. Randomized comparison of mefloquine-artesunate versus quinine in the treatment of multidrug-resistant falciparum malaria in pregnancy. Trans R Soc Trop Med Hyg 2000;94:689-93.

† PCR-adjusted.

$\S$ McGready R, Cho T, Keo NK, et al. Artemisinin antimalarials in pregnancy: a prospective treatment study of 539 episodes of multidrug-resistant Plasmodium falciparum. Clin Infect Dis 2001;33:2009-16.

"McGready R, Ashley EA, Moo E, et al. A randomized comparison of artesunate-atovaquone-proguanil versus quinine in treatment for uncomplicated falciparum malaria during pregnancy. J Infect Dis 2005;192:846-53.

** 37 of 39 participants.

†† 26 of 41 participants.

$\S \S$ Piola P, Nabasumba C, Turyakira E, et al. Efficacy and safety of artemether-lumefantrine compared with quinine in pregnant women with uncomplicated Plasmodium falciparum malaria: an open-label, randomised, non-inferiority trial. Lancet Infect Dis 2010;10:762-9.

กी 137 of 139 participants.

*** 122 of 125 participants.

${ }^{+t \dagger}$ Kaye DK, Nshemerirwe R, Mutyaba TS, Ndeezi G. A randomized clinical trial comparing safety, clinical and parasitological response to artemether-lumefantrine and chlorproguanil-dapsone in treatment of uncomplicated malaria in pregnancy in Mulago hospital, Uganda. J Infect Dev Ctries 2008;2:135-9.

women taking ACTs versus non-ACTs to treat uncomplicated malaria in the second and third trimesters of pregnancy (pooled risk ratio random effects $=0.41 ; 95 \%$ confidence interval $(\mathrm{CI})$ $=0.16-1.06$; six trials) (5). With respect to AL efficacy during the second and third trimesters of pregnancy, a concern existed that a reduction in relative bioavailability of lumefantrine in pregnant women might affect treatment success later in pregnancy (11-15). However, the evidence presented indicates that treatment in pregnancy is efficacious at the doses currently recommended for nonpregnant women.

Second and third trimester safety. Data evaluating pregnancy outcomes in women taking ACTs during the second or third trimesters of pregnancy were available from 16 studies (Table 2). No differences in pregnancy outcomes were identified in four trials comparing ACTs with quinine-based regimens in Uganda and Thailand $(6,7,9,10)$, one of which used AL (9), and in four other trials comparing AL with other ACTs in Nigeria (two studies), Thailand, and multiple sites in Africa (16-19). A Zambian cohort study comparing treatment of uncomplicated malaria using AL with treatment using sulfadoxine-pyrimethamine found similar pregnancy outcomes between groups (20). In addition, two meta-analyses of women with malaria in the second and third trimester of pregnancy found no association between ACT treatment and congenital malformations or miscarriage $(5,21)$. Overall, fewer maternal adverse events occurred among women taking ACTs than among those taking non-ACTs (Table 2). One trial in Thailand found a relatively higher proportion of day 7 anemia among those treated with mefloquine-artesunate (67\%) than among those treated with a quinine-based regimen (42\%) (6). Four trials and one meta-analysis comparing ACTs with quinine-based regimens found that pregnant women taking quinine had higher rates of tinnitus, dizziness, and vomiting than did pregnant women taking ACTs (5-9). The three trials comparing AL with other ACTs found no differences in rates of serious adverse maternal effects between groups $(9,16,18)$.

First trimester safety. No randomized trials evaluating AL use during the first trimester of pregnancy were found (Table 3). However, a meta-analysis of observational and other studies from six sub-Saharan African countries and the Thai-Burmese border included data from a total of 717 women taking ACTs during the first trimester of pregnancy (22). Comparisons of pregnancy outcomes between women taking ACTs and those receiving a quinine-based regimen 
TABLE 2. Summary of studies using artemisinin-based treatment for malaria in second and third trimesters of pregnancy and safety outcomes

\begin{tabular}{|c|c|c|c|c|c|c|}
\hline $\begin{array}{l}\text { Author, } \\
\text { publication year }\end{array}$ & Indication (country) & Drug(s) & $\begin{array}{c}\text { No. of } \\
\text { participants }\end{array}$ & $\begin{array}{c}\text { Pregnancy outcomes, } \\
\mathrm{n} / \mathrm{N}(\%)^{*}\end{array}$ & $\begin{array}{c}\text { Congenital } \\
\text { anomalies, n/N (\%) }\end{array}$ & $\begin{array}{c}\text { Maternal adverse } \\
\text { events, } \mathrm{n} / \mathrm{N}(\%)\end{array}$ \\
\hline \multicolumn{7}{|c|}{ Randomized trials (all open label) using nonartemisinin drug in comparison group } \\
\hline \multirow[t]{2}{*}{$\begin{array}{l}\text { McGready, et al., } \\
2000^{\dagger}\end{array}$} & \multirow[t]{2}{*}{$\begin{array}{l}\text { Uncomplicated } \\
\text { P. falciparum } \\
\text { (Thailand) }\end{array}$} & $\begin{array}{l}\text { MQ } 25 \mathrm{mg} / \mathrm{kg} \times 1 \text { and As } \\
4 \mathrm{mg} / \mathrm{kg} / \mathrm{d} \times 3 \mathrm{~d}\end{array}$ & 66 & $\begin{array}{l}\text { Miscarriage, } 2(3) \\
\text { Stillbirth, } 0(0) \\
\text { Low birth weight, } \\
\text { 9/53 (17) }\end{array}$ & $0(0)$ & $\begin{array}{l}\text { Anemia day } 7,32 / 48(67)^{\S} \\
\text { Dizziness, }(45)^{\S} \\
\text { Tinnitus, }(17)^{\S}\end{array}$ \\
\hline & & Q $10 \mathrm{mg} / \mathrm{kg}$ q8hr $\times 7 \mathrm{~d}$ & 42 & $\begin{array}{l}\text { Miscarriage, } 0(0) \\
\text { Stillbirths, } 0(0) \\
\text { Low birth weight, } \\
6 / 33(18)\end{array}$ & $0(0)$ & $\begin{array}{l}\text { Anemia day } 7,14 / 33(42)^{\S} \\
\text { Dizziness, }(87)^{\S} \\
\text { Tinnitus, }(66)^{\S}\end{array}$ \\
\hline $\begin{array}{l}\text { McGready, et al., } \\
20019\end{array}$ & $\begin{array}{l}\text { Uncomplicated } \\
\text { P. falciparum } \\
\text { (Thailand) }\end{array}$ & $\begin{array}{l}\text { As } 2 \mathrm{mg} / \mathrm{kg} / \mathrm{d} \times 7 \mathrm{~d} \\
\mathrm{Q} 10 \mathrm{mg} / \mathrm{kg} \mathrm{q} 8 \mathrm{hr} \times 7 \mathrm{~d} \text { and } \\
\text { CL } 5 \mathrm{mg} / \mathrm{kg} \mathrm{q} 8 \mathrm{hr} \times 7 \mathrm{~d}\end{array}$ & $\begin{array}{l}64 \\
65\end{array}$ & $\begin{array}{l}\text { Stillbirth, } 1(2)^{* *} \\
\text { Stillbirth, } 1(2)^{* *}\end{array}$ & $\begin{array}{l}\text { Minor, } 1(2) \\
\text { Major, } 1(2)\end{array}$ & $\begin{array}{l}\text { Tinnitus, }(9)^{\S} \\
\text { Tinnitus, }(45)^{\S}\end{array}$ \\
\hline \multirow[t]{2}{*}{$\begin{array}{l}\text { McGready, et al., } \\
2005^{+t, \S \S}\end{array}$} & \multirow[t]{2}{*}{$\begin{array}{l}\text { Uncomplicated } \\
\text { P. falciparum } \\
\text { (Thailand) }\end{array}$} & $\begin{array}{l}\text { As } 4 \mathrm{mg} / \mathrm{kg} / \mathrm{d} \times 3 \mathrm{~d} \text { and } A \\
20 \mathrm{mg} / \mathrm{kg} / \mathrm{d} \times 3 \mathrm{~d} \text { and } \\
\text { P } 8 \mathrm{mg} / \mathrm{kg} / \mathrm{d} \times 3 \mathrm{~d}\end{array}$ & 39 & $\begin{array}{l}\text { Preterm, } 4 / 34(12) \\
\text { Low birth weight, } \\
6 / 23(26)\end{array}$ & $\begin{array}{l}\text { Polythelia and cleft lip } \\
\text { and palate, } 2 / 34(6)^{* *}\end{array}$ & Tinnitus, $(24)^{\S}$ \\
\hline & & $\mathrm{Q} 10 \mathrm{mg} / \mathrm{kg} \mathrm{q} 8 \mathrm{hr} \times 7 \mathrm{~d}$ & 42 & $\begin{array}{l}\text { Stillbirth, } 1 \text { (2) } \\
\text { Preterm, 6/38 (16) } \\
\text { Low birth weight, } \\
4 / 30(13)\end{array}$ & $\begin{array}{l}\text { Left aural atresia, } \\
1 / 38(3)^{* *}\end{array}$ & Tinnitus, $(79)^{\S}$ \\
\hline \multirow[t]{8}{*}{$\begin{array}{l}\text { Piola, et al., } \\
2010 \text { १ी }\end{array}$} & \multirow[t]{8}{*}{$\begin{array}{l}\text { Uncomplicated } \\
\text { P. falciparum } \\
\text { (Uganda) }\end{array}$} & \multirow[t]{4}{*}{$\begin{array}{l}\text { AL } 20 / 120 \mathrm{mg} 4 \text { tabs at } 0 \\
\text { and } 8 \mathrm{hr} \times 1 \mathrm{~d} \text {, then BID } \times 2 \mathrm{~d}\end{array}$} & \multirow[t]{4}{*}{152} & $\begin{array}{l}\text { Miscarriage, } 2 / 144(1) \\
\text { Intrauterine fetal } \\
\text { death, } 1 / 144(1)\end{array}$ & \multirow[t]{4}{*}{$\begin{array}{l}\text { Polydactyly, } 2(1)^{* *} \\
\text { Acyanotic heart } \\
\text { disease, } 1(1)\end{array}$} & $\begin{array}{l}\text { Tinnitus, } 0(0)^{\S} \\
\text { Headache, } 26(17)^{\S}\end{array}$ \\
\hline & & & & Stillbirth, 2/144 (1) & & Nausea, $8(5)^{\S}$ \\
\hline & & & & Preterm, 12/143 (1) & & Vomiting, $6(4)^{\S}$ \\
\hline & & & & $\begin{array}{l}\text { Low birth weight, } \\
12 / 120(10)\end{array}$ & & Anorexia, $6(4)^{\S}$ \\
\hline & & \multirow[t]{4}{*}{ Q $10 \mathrm{mg} / \mathrm{kg}$ q8hr $\times 7 \mathrm{~d}$} & \multirow[t]{4}{*}{152} & $\begin{array}{l}\text { Miscarriage, } 2 / 137 \text { (2) } \\
\text { Intrauterine fetal } \\
\text { death, } 2 / 137(2)\end{array}$ & \multirow[t]{4}{*}{ Polydactyly, 2 (1)** } & $\begin{array}{l}\text { Tinnitus, } 111(73)^{\S} \\
\text { Headache, } 9(6)^{\S}\end{array}$ \\
\hline & & & & Stillbirth, 3/137 (2) & & Nausea, $26(17)^{\S}$ \\
\hline & & & & Preterm, $17 / 137(3)$ & & Vomiting, $28(18)^{\S}$ \\
\hline & & & & $\begin{array}{l}\text { Low birth weight, } \\
16 / 137(13)\end{array}$ & & Anorexia, $16(11)^{\S}$ \\
\hline \multirow[t]{2}{*}{$\begin{array}{l}\text { Kaye, et al., } \\
2008^{* * *}\end{array}$} & \multirow[t]{2}{*}{$\begin{array}{l}\text { Uncomplicated } \\
\text { P. falciparum } \\
\text { (Uganda) }\end{array}$} & $\begin{array}{l}\text { AL } 20 / 120 \mathrm{mg} 4 \text { tabs at } 0 \\
\text { and } 8 \mathrm{hr} \times 1 \mathrm{~d} \text {, then BID } \times 2 \mathrm{~d}\end{array}$ & 57 & Not assessed & Not assessed & $\begin{array}{l}\text { Palpitations, } 4 \text { (7) } \\
\text { Dizziness, } 1 \text { (2) } \\
\text { Drowsiness, } 1 \text { (2) } \\
\text { Rash, } 1 \text { (2) }\end{array}$ \\
\hline & & LapDap x 3d & 57 & Not assessed & Not assessed & $\begin{array}{l}\text { Vomiting, } 1 \text { (2) } \\
\text { Diarrhea, } 1 \text { (2) } \\
\text { Palpitations, } 1 \text { (2) }\end{array}$ \\
\hline Randomized trial & s (open label unless or & therwise noted) using artemis & in in comparis & ison group & & \\
\hline $\begin{array}{l}\text { Sowunmi, et al., } \\
1998^{t+t}\end{array}$ & $\begin{array}{l}\text { Failed CQ, SP or } \\
\text { CQ-SP treatment }\end{array}$ & $\begin{array}{c}\text { Ar } 3.2 \mathrm{mg} / \mathrm{kg} \mathrm{IM} \times 1 \text { then } \\
1.6 \mathrm{mg} / \mathrm{kg} \mathrm{IM} \mathrm{qd} \mathrm{x} \mathrm{4d}\end{array}$ & 23 & & None & None \\
\hline & $\begin{array}{l}\text { for P. falciparum } \\
\text { (Nigeria) }\end{array}$ & $\begin{array}{l}\text { Ar } 3.2 \mathrm{mg} / \mathrm{kg} \text { IM } \times 1 \text { then } \\
\text { MQ } 7.5 \mathrm{mg} / \mathrm{kg} \text { qd } \times 2 \mathrm{~d}\end{array}$ & 22 & None & None & $\begin{array}{l}\text { Abdominal discomfort, } \\
2(9) \\
\text { Dizziness, } 2 \text { (9) }\end{array}$ \\
\hline 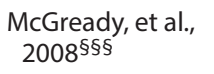 & $\begin{array}{l}\text { Uncomplicated } \\
\text { P. falciparum }\end{array}$ & $\begin{array}{l}\text { AL 20/120 mg } 4 \text { tabs } \\
\text { BID } \times 3 d\end{array}$ & 124 & $\begin{array}{l}\text { Miscarriage, } 0(0) \\
\text { Stillbirth, } 1 / 119(1)\end{array}$ & None & Vomiting, 2 (2) \\
\hline & (Thailand) & As $2 \mathrm{mg} / \mathrm{kg} \mathrm{qd} \times 7 \mathrm{~d}$ & 125 & $\begin{array}{l}\text { Miscarriage, } 1 / 122(1)^{* *} \\
\text { Stillbirth, } 1 / 119(1)\end{array}$ & None & $\begin{array}{l}\text { Vomiting, } 1 \text { (1) } \\
\text { Rash, } 1 \text { (1) }\end{array}$ \\
\hline $\begin{array}{l}\text { Ukah, et al., } \\
2015 \text { ๆศศศ }\end{array}$ & $\begin{array}{l}\text { Uncomplicated } \\
\text { P. falciparum }\end{array}$ & $\mathrm{AL}(80 \mathrm{mg} / 480 \mathrm{mg}) \mathrm{BID} \times 3 \mathrm{~d}$ & 75 & $\begin{array}{l}\text { Miscarriage, 1/71 (1) } \\
\text { Stillbirth, 2/71 (3) }\end{array}$ & Not assessed & $\begin{array}{l}\text { Body weakness } 2(3) \\
\text { Pruritis } 0(0)\end{array}$ \\
\hline & $\begin{array}{l}\text { (Nigeria, } \\
\text { double-blind) }\end{array}$ & $\begin{array}{l}\text { Ar-AQ (100 mg/270 mg) } \\
\text { BID x 3d }\end{array}$ & 75 & $\begin{array}{l}\text { Miscarriage, 1/65 (2) } \\
\text { Stillbirth, 1/65 (2) }\end{array}$ & Not assessed & $\begin{array}{l}\text { Body weakness, } 26 \text { (35) } \\
\text { Pruritis, } 4 \text { (5) }\end{array}$ \\
\hline
\end{tabular}

See table footnotes on page 428. 
TABLE 2. (Continued) Summary of studies using artemisinin-based treatment for malaria in second and third trimesters of pregnancy and safety outcomes

\begin{tabular}{|c|c|c|c|c|c|c|}
\hline $\begin{array}{l}\text { Author, } \\
\text { publication year }\end{array}$ & Indication (country) & Drug(s) & $\begin{array}{c}\text { No. of } \\
\text { participants }\end{array}$ & $\begin{array}{c}\text { Pregnancy outcomes, } \\
\mathrm{n} / \mathrm{N}(\%)^{*}\end{array}$ & $\begin{array}{c}\text { Congenital } \\
\text { anomalies, } \mathrm{n} / \mathrm{N}(\%)\end{array}$ & $\begin{array}{c}\text { Maternal adverse } \\
\text { events, } \mathrm{n} / \mathrm{N}(\%)\end{array}$ \\
\hline \multirow[t]{4}{*}{$\begin{array}{l}\text { PREGACT, } \\
2016^{* * * *}\end{array}$} & \multirow[t]{4}{*}{$\begin{array}{l}\text { P. falciparum } \\
\text { (four African } \\
\text { countries) }\end{array}$} & $\mathrm{AL}$ & 880 & $\begin{array}{l}\text { Miscarriage, } 1 \\
\text { Stillbirth, 16/856 (2) } \\
\text { Preterm, (10) }\end{array}$ & Any defect, $17 / 832(2)$ & \\
\hline & & AQ-As & 842 & $\begin{array}{l}\text { Miscarriage, } 4(<1) \\
\text { Stillbirth, 17/815 (2) } \\
\text { Preterm, (3) }\end{array}$ & Any defect, 8/776 (1) & $\begin{array}{l}\text { Anemia, } 2(<1 \\
\text { Abdominal pain, } 1(<1) \\
\text { Malaise, } 2(<1)\end{array}$ \\
\hline & & MQ-As & 848 & $\begin{array}{l}\text { Miscarriage, } 4 \\
\text { Stillbirth, 23/821 (3) } \\
\text { Preterm, (8) }\end{array}$ & Any defect, $13 / 780(2)$ & $\begin{array}{l}\text { Abdominal pain, } 1(<1) \\
\text { Vomiting, } 2(<1) \\
\text { Malaise, } 1(<1)\end{array}$ \\
\hline & & DHA-PIP & 853 & $\begin{array}{l}\text { Miscarriage, } 4(<1) \\
\text { Stillbirth, 22/818 (3) } \\
\text { Preterm, (10) }\end{array}$ & Any defect, 6/767 (1) & Headache/weakness, $1(<1)$ \\
\hline \multicolumn{7}{|l|}{ Cohort study } \\
\hline \multirow[t]{2}{*}{ 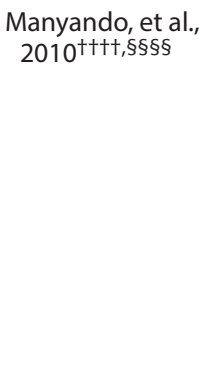 } & \multirow[t]{2}{*}{$\begin{array}{l}\text { Uncomplicated } \\
\text { P. falciparum } \\
\text { (Zambia) }\end{array}$} & $\begin{array}{l}\text { AL } 20 \mathrm{mg} / 120 \mathrm{mg} 4 \text { tabs } \\
\text { BID } \times 3 \mathrm{~d}\end{array}$ & 495 & $\begin{array}{l}\text { Miscarriage, } 7 / 504 \text { (1) } \\
\text { (all first trimester } \\
\text { exposures) } \\
\text { Stillbirth, 9/504 (2) } \\
\text { Preterm, 71/504 (14) }\end{array}$ & Any defect, $29 / 449(7)$ & Not reported \\
\hline & & $\mathrm{SP}(1500 \mathrm{mg} / 75 \mathrm{mg})$ & 506 & $\begin{array}{l}\text { Miscarriage, 8/516 (2) } \\
\text { (in } 5 \text { women, } \\
\text { including one with } \\
\text { twins and one with } \\
\text { triplets) } \\
\text { Stillbirth, 13/516 (3) } \\
\text { Preterm, 90/516 (17) }\end{array}$ & Any defect, 18/444 (4) & Not reported \\
\hline \multicolumn{7}{|c|}{ Descriptive studies (includes pharmacokinetic studies and case series) } \\
\hline \multirow[t]{2}{*}{$\begin{array}{l}\text { McGready, et al., } \\
2001 \text { (includes } \\
\text { data published } \\
\text { 1998) }\end{array}$} & \multirow[t]{2}{*}{$\begin{array}{l}\text { P. falciparum or } \\
\text { mixed, primary } \\
\text { and recrudescent, } \\
\text { uncomplicated } \\
\text { and severe } \\
\text { (Thailand) }\end{array}$} & $\begin{array}{l}\text { As given } 2-4 \mathrm{mg} / \mathrm{kg} \text { up to } \\
7 \text { days (varies by } \\
\text { indication) or As } 4 \mathrm{mg} / \mathrm{kg} \\
\mathrm{qd} \times 3 \mathrm{~d} \text { and } \mathrm{AP} \text { or } \mathrm{As} \\
4 \mathrm{mg} / \mathrm{kg} \text { qd } \times 3 \mathrm{~d} \text { and } \mathrm{MQ}\end{array}$ & 461 & $\begin{array}{l}\text { Miscarriage, 20/414 (5) } \\
\text { Stillbirth, 7/386 (2) }\end{array}$ & $\begin{array}{l}\text { Any defect, } 3 / 386(1) \\
\text { Major } 1 / 386(0)\end{array}$ & $\begin{array}{l}\text { No serious adverse } \\
\text { events }\end{array}$ \\
\hline & & Community (no treatment) & & $\begin{array}{l}\text { Miscarriage, } \\
1003 / 8154(12) \\
\text { Stillbirth, } 114 / 7058(2) \\
\text { Low birth weight, } \\
866 / 6418(14)\end{array}$ & Any defect, $866 / 6418(14)$ & \\
\hline $\begin{array}{l}\text { Mosha, et al., } \\
2014^{* * * * * *}\end{array}$ & $\begin{array}{l}\text { Uncomplicated } \\
\text { P. falciparum } \\
\text { (Tanzania) }\end{array}$ & $\begin{array}{l}\text { AL } 20 / 120 \mathrm{mg} 4 \text { tabs at } 0 \\
\text { and } 8 \mathrm{hr} \times 1 \mathrm{~d} \text {, then BID } \times 2 \mathrm{~d}\end{array}$ & 35 & $\begin{array}{l}\text { Not assessed, } \\
\text { (follow-up to } \\
42 \text { days only) }\end{array}$ & Not assessed & $\begin{array}{l}\text { No serious adverse } \\
\text { events }\end{array}$ \\
\hline $\begin{array}{l}\text { Nyunt, et al., } \\
2015^{++t+\dagger}\end{array}$ & $\begin{array}{l}\text { Uncomplicated } \\
\text { P. falciparum } \\
\text { (Uganda) }\end{array}$ & $\begin{array}{l}\mathrm{AL} 20 / 120 \mathrm{mg} 4 \text { tabs at } 0 \\
\text { and } 8 \mathrm{hr} \times 1 \mathrm{~d} \text {, then BID } \times 2 \mathrm{~d}\end{array}$ & 30 & $\begin{array}{l}\text { Not assessed, } \\
\text { (follow-up to } \\
42 \text { days only) }\end{array}$ & Not assessed & $\begin{array}{l}\text { No serious adverse } \\
\text { events }\end{array}$ \\
\hline 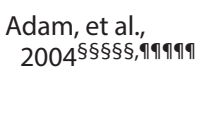 & $\begin{array}{l}\text { P. falciparum } \\
\text { (Sudan) }\end{array}$ & $\begin{array}{l}\operatorname{Ar} 80 \mathrm{mg} \text { IM BID x } 1 \mathrm{~d} \text { then } \\
\mathrm{qd} \times 2 \mathrm{~d}\end{array}$ & 28 & $\begin{array}{l}\text { Miscarriage, } 0(0) \\
\text { Stillbirth, } 0(0) \\
\text { Premature, } 1(4)^{* *}\end{array}$ & Not assessed & Not assessed \\
\hline 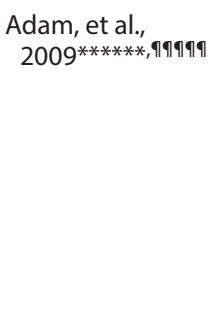 & $\begin{array}{l}\text { P. falciparum } \\
\text { (Sudan) }\end{array}$ & $\begin{array}{l}\text { Ar IM } \\
\text { As and SP } \\
\text { AL }\end{array}$ & 62 & $\begin{array}{l}\text { Miscarriage, } 2(3)^{* *} \\
\text { (both had received } \\
\text { artemether } \\
\text { injections early in } \\
\text { pregnancy and } \\
\text { miscarried while } \\
\text { receiving quinine } \\
\text { infusions for a } \\
\text { second malaria } \\
\text { infection) }\end{array}$ & Not assessed & Not assessed \\
\hline Wang, $1981^{\text {tttttt }}$ & $\begin{array}{l}\text { "Plasmodium" } \\
\text { (China) }\end{array}$ & $\begin{array}{l}\text { Ar in oil } 500-900 \mathrm{mg} \mathrm{IM} \mathrm{qd} \mathrm{x} \\
3 \mathrm{~d} \text { or Ar } 600 \mathrm{mg} \text { IM qd x 3d }\end{array}$ & 6 & $\begin{array}{l}\text { Miscarriage, } 0(0) \\
\text { Stillbirth, } 0(0) \\
\text { Premature, } 0(0)\end{array}$ & Any defect, $0(0)$ & Not assessed \\
\hline
\end{tabular}

See table footnotes on page 428. 


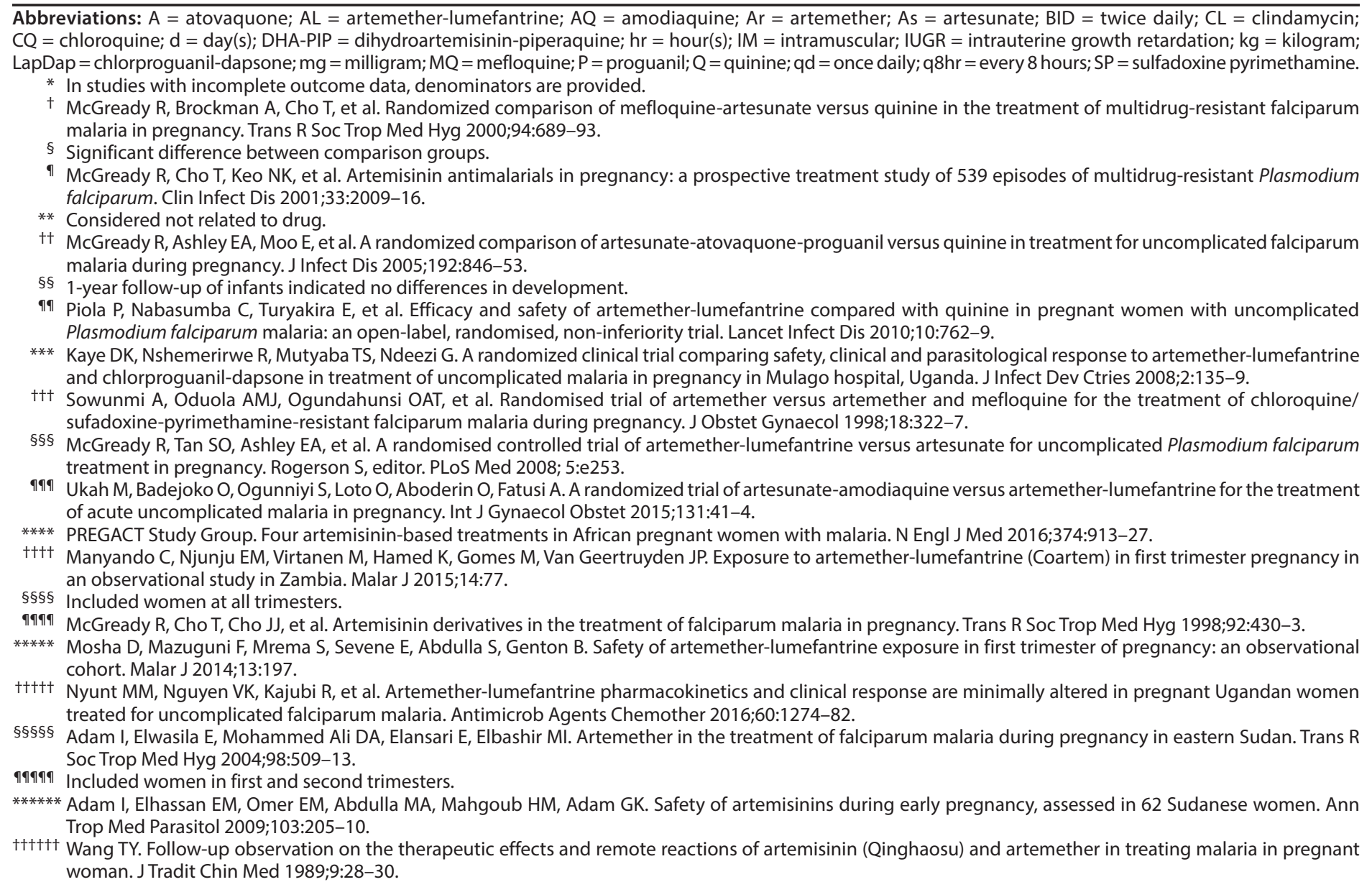

anytime during the first trimester and treatment with ACTs versus quinine-based regimen during 6-12 weeks' gestational age demonstrated no differences in miscarriage, stillbirth, or pregnancy loss (miscarriage and still birth combined) for women treated with ACTs versus quinine-based regimens during either period. Although limited by sample size, the pooled prevalences of congenital anomalies in infants born to mothers taking ACTs versus quinine-based regimens in the first trimester were similar $(1.5 \%, 95 \% \mathrm{CI}=0.6-3.5$ versus $1.2 \%, 95 \% \mathrm{CI}=0.6-2.4$, respectively) (22).

\section{Recommendation}

Malaria infection during pregnancy can result in serious maternal and fetal complications. On the basis of the strength and quality of this evidence, CDC recommends $\mathrm{AL}$ as an additional option for treatment of uncomplicated malaria in pregnant women in the United States during the second and third trimesters of pregnancy at the same doses recommended for nonpregnant women. Women in the United States with uncomplicated malaria during the first trimester of pregnancy should be treated with the currently recommended options of either mefloquine or quinine plus clindamycin. However, when neither of these options is available, AL should be considered for treatment.

\section{Discussion}

This update of CDC recommendations based on accumulated evidence of the safety of AL in pregnancy is in line with the malaria treatment guidelines of other countries without endemic malaria and $\operatorname{WHO}(3,23,24)$. On the basis of the current strength and quality of the first trimester safety and efficacy evidence, the addition of ACTs, including AL, as a first-line treatment option for uncomplicated malaria during the first trimester of pregnancy is being considered by WHO after the Malaria Policy Advisory Committee's review $(2,3)$. Women seeking care in the United States will now have a third treatment option for uncomplicated malaria during the second and third trimesters of pregnancy, and during the first trimester of pregnancy when other treatment options are unavailable, that is safe and effective for treating $P$. falciparum infections acquired in regions with chloroquine resistance. To assess the implementation and impact of these updated recommendations in the United States, data from the National Malaria Surveillance System will be used to examine how antimalarials 
Author,

Description or indication (country)

Meta-analysis
Dellicour, et al. $2017^{\dagger}$

Included five observational studies (individual participant data from six sub-Saharan African countries, and aggregate data from Thailand)

\section{Observational studies} Any anomaly, 1 (1) inadvertent use of AL, other Any anomaly, 2 (3) antimalarials, or none, then followed to birth outcome (Tanzania)

Dellicour, et al. $2015^{* *, 9}$
Identified women with inadvertent use of $\mathrm{AL}$, other antimalarials, or none, then followed to birth outcome (Kenya)
Drug or

regimen (no.) no. (\%) (unless otherwise indicated)*
Pregnancy outcomes,
Congenital anomalies, no. (\%) (unless otherwise indicated) o. (\%) (unless otherwise indicated)

$\begin{array}{ll}\text { Areg (717) } & \text { Miscarriage: } \\ & \text { Areg versus Q: } \mathrm{aHR}=0.73(95 \% \mathrm{Cl}=0.44-1.21) \\ & \text { Areg versus none: } \mathrm{aHR}=1.16(95 \% \mathrm{Cl}=0.81-1.66) \\ \mathrm{Q}(947) & \text { Stillbirth: } \\ & \text { Areg versus Q: } \mathrm{aHR}=0.29(95 \% \mathrm{Cl}=0.08-1.02) \\ & \text { Areg versus none: } \mathrm{aHR}=0.65(95 \% \mathrm{Cl}=0.34-1.23) \\ \text { No antimalarials } & \text { Stillbirth and miscarriage: } \\ (28,954) & \text { Areg versus Q: } \text { aHR }=0.58(95 \% \mathrm{Cl}=0.36-1.02)\end{array}$

$A L(164)$

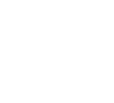

$\mathrm{Q}(70)$

SP (66)
Miscarriage, 3(4.3) and stillbirth, 5 (7.1);
$\mathrm{aOR}=2.5(95 \% \mathrm{Cl}=1.3-5.1, \mathrm{p}=0.009)$

Low birth weight, 1 (1.6);

$\mathrm{aOR}=0.6(0.1-2.4, \mathrm{p}=0.461)$

$\mathrm{aOR}=1.4(95 \% \mathrm{Cl}=0.8-2.5, \mathrm{p}=0.295)$

Low birth weight, 8 (5.2);

Preterm, 8 (5.2);

aOR $=0.9(95 \% \mathrm{Cl}=0.5-1.8, \mathrm{p}=0.865)$

Preterm, aOR $=2.6(95 \% \mathrm{Cl}=1.3-5.3, \mathrm{p}=0.007)$
As $1.5 \%(95 \%$
$\mathrm{Cl}=0.6-3.5) ; \mathrm{Q} 1.2 \%$
$(95 \% \mathrm{Cl}=0.6-2.4)$

Miscarriage, 0 and stillbirth, 2 (3.0);
$\mathrm{aOR}=1.2(95 \% \mathrm{Cl}=0.6-2.5, \mathrm{p}=0.573)$

Not assessed

$\mathrm{aOR}=0.5(95 \% \mathrm{Cl}=0.1-2.0, \mathrm{p}=0.312)$

Low birth weight, 2 (3.1);

$\mathrm{aOR}=0.7(95 \% \mathrm{Cl}=0.2-3.0, \mathrm{p}=0.639)$

Preterm, 7 (10.9):

AQ (11) $\quad \mathrm{aOR}=1.8(95 \% \mathrm{Cl}=0.8-4.1, \mathrm{p}=0.160)$

Miscarriage, 0 and stillbirth, 0

Low birth weight, 0

Preterm, 0

No antimalarials Miscarriage, 34 (2.3) and stillbirth, 49 (3.3);

$(1,464) \quad \mathrm{aOR}=0.8(95 \% \mathrm{Cl}=0.5-1.2, \mathrm{p}=0.260)$

Low birth weight, 69 (5.0);

$\mathrm{aOR}=1.2(95 \% \mathrm{Cl}=0.6-2.3, \mathrm{p}=0.564)$

Preterm, 88 (6.4);

$\mathrm{aOR}=0.7(95 \% \mathrm{Cl}=0.5-1.1, \mathrm{p}=0.168)$

Confirmed ACT Miscarriage:

(77)

Unconfirmed Confirmed ACT exposure only:

ACT (222) ACT 6/77 versus no antimalarial 57/793

$\mathrm{Q}$ (13) $\quad \mathrm{aHR}=1.72(95 \% \mathrm{Cl}=0.66-4.45, \mathrm{p}=0.266)$

No ACT

exposure

$\mathrm{Q} 0 / 3$ versus no antimalarial $57 / 793$

(835)

ACT 5/72 versus Q 1/13;

$\mathrm{aHR}=0.48(95 \% \mathrm{Cl}=0.12-1.89, \mathrm{p}=0.297)$

Confirmed and unconfirmed ACT: ACT 29/299

versus no antimalarial 57/793;

$\mathrm{aHR}=1.66(95 \% \mathrm{Cl}=1.04-2.67, \mathrm{p}=0.034)$

$Q$ 1/13 versus no antimalarial 57/793;

$\mathrm{aHR}=4.27(95 \% \mathrm{Cl}=0.53-34.33, \mathrm{p}=0.172)$

ACT 28/286 versus Q 1/13;

$\mathrm{aHR}=0.64(95 \% \mathrm{Cl}=0.08-4.91, \mathrm{p}=0.665)$

Moore, et al.
$2016^{t+, 9}$

Data from antenatal clinics analyzed (Thai-Myanmar border)

Areg (183)

Miscarriage: when compared with $\mathrm{Q}$ or $\mathrm{Q}$ and $\mathrm{CL}$
Areg, $92(11): \mathrm{aHR}=0.78(95 \% \mathrm{Cl}=0.45-1.34$, $\mathrm{p}=0.3645)$

MQ (25)

MQ 2 (8): $a H R=0.54(95 \% \mathrm{Cl}=0.13-2.31, \mathrm{p}=$ $0.4082)$

$\mathrm{Q}$ or $\mathrm{Q}$ and $\mathrm{CL}$ When comparing malaria with no malaria in first (971) trimester, miscarriage: $\mathrm{aHR}=1.61(95 \% \mathrm{Cl}=$ $1.32-1.97, p<0.0001$ )
Any malformation: Uncomplicated Pf treated with Areg, 2/109 (2), Q, 9/641 (1), Severe Pf treated with: Areg, 2/22 (9); Q, $0 / 8(0)$
Not assessed

Not assessed

Not assessed

Not assessed

Not assessed

Not assessed

Not assessed

\footnotetext{
See table footnotes on page 430 .
} 
Author,

publication year

Description or indication (country)

Manyando, et al., $2015^{\S \S, 9}$

Data analyzed from previous prospective cohort, women with inadvertent first trimester exposure (Zambia)

$\mathrm{AL}$ and SP (7)

SP and/or $\mathrm{Q}$

No antimalarial (644) no. (\%) (unless otherwise indicated)*
Congenital anomalies,

Miscarriage not assessed Stillbirth, 2 (1.5) $(95 \% \mathrm{Cl}=0.4-5.2)$ Low birth weight, 13 (10.2)

Miscarriage not assessed Stillbirth, 0 (0) $(95 \% \mathrm{Cl}=0-39.0)$ Low birth weight, 1 (14.3)

Miscarriage not assessed Stillbirth, 3 (2.3) $(95 \% \mathrm{Cl}=0.8-6.6)$ Low birth weight, 8 (6.7) Stillbirth, 17 (2.6) $(95 \% \mathrm{Cl}=1.7-4.2)$ Low birth weight, 52 (8.7)

\section{Descriptive studies}

McGready, et al.,

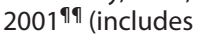
data from McGready et al. 1998)***
P for mixed infection, both primary and recrudescent, uncomplicated and severe (Thailand)
Areg (19 Miscarriage, 7 (18.9) $)^{\text {t+† }}$

primary treat-

ment, 25 for

retreatment)

Community (no Miscarriage, 1,003/8,154 (12.3) treatment) no. (\%) (unless otherwise indicated)

Any malformation,

9 (7)

Any malformation, Not assessed 8/121 (7) otherwise indicated)

Not assessed

Not assessed

Not assessed

Not assessed

Any malformation,

Not assessed 0

Any malformation, Not assessed $56 / 3,707$ (2)

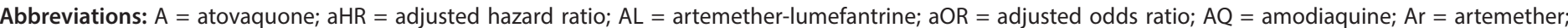

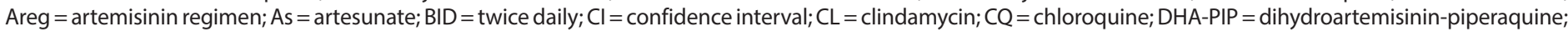
LapDap = chlorproguanil-dapsone; $\mathrm{MQ}=$ mefloquine; $\mathrm{P}=$ proguanil; $\mathrm{Pf}=$ Plasmodium falciparum; $\mathrm{Q}=$ quinine; $\mathrm{SP}=$ sulfadoxine $\mathrm{pyrimethamine}$

* In studies with incomplete outcome data, denominators are provided.

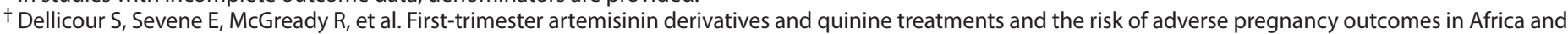
Asia: a meta-analysis of observational studies. Krishna S, editor. PLOS Med 2017;14:e1002290.

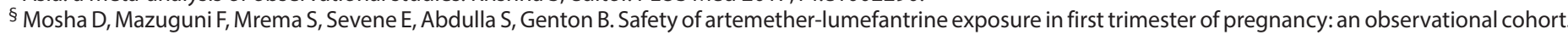
Malar J 2014;13:197.

I Study included in Dellicour 2017 meta-analysis.

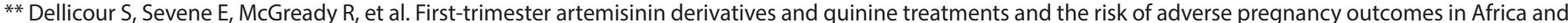
Asia: a meta-analysis of observational studies. Krishna S, editor. PLOS Med 2017;14:e1002290.

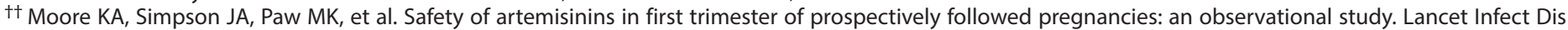
2016;16:576-83.

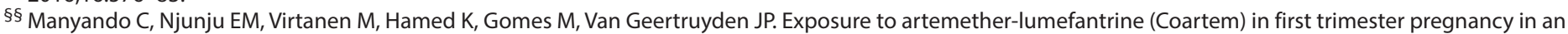
observational study in Zambia. Malar J 2015;14:77.

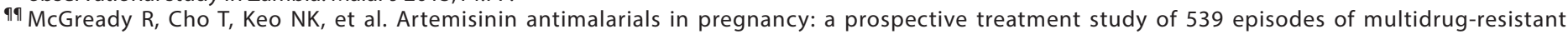
Plasmodium falciparum.

*** McGready R, Cho T, Cho JJ, et al. Artemisinin derivatives in the treatment of falciparum malaria in pregnancy. Trans R Soc Trop Med Hyg 1998;92:430-3.

${ }^{+\dagger \dagger}$ Not different from overall community rate $\mathrm{p}=0.211$

are used to treat uncomplicated malaria in pregnant women, as well as population-specific disease burden; in addition, the FDA Adverse Event Reporting System maintains adverse event and medication error data, which can be used to monitor adverse events associated with AL use during pregnancy.

\section{Conflict of Interest}

No conflicts of interest were reported.

${ }^{1}$ Epidemic Intelligence Service, CDC; ${ }^{2}$ Division of Parasitic Diseases and Malaria, Center for Global Health, CDC; ${ }^{3}$ Hubert Department of Global Health, Emory University, Atlanta, Georgia.

Corresponding author: Sarah-Blythe Ballard, sballard2@cdc.gov, 404-718-6711

\section{References}

1. CDC. Guidelines for treatment of malaria in the United States. Atlanta, GA: US Department of Health and Human Services, CDC; 2013. https://www.cdc.gov/malaria/resources/pdf/treatmenttable.pdf
2. World Health Organization Malaria Policy Advisory Committee. Intermittent screening and treatment in pregnancy and the safety of ACTs in the first trimester. Geneva, Switzerland: World Health Organization; 2015. http://www.who.int/malaria/publications/atoz/ istp-and-act-in-pregnancy.pdf?ua $=1$

3. World Health Organization. Guidelines for the treatment of malaria. 3rd ed. Geneva, Switzerland: World Health Organization; 2015.

4. Mace KE, Arguin PM. Malaria surveillance-United States, 2014. MMWR Surveill Summ 2017;66 (No. SS-12). https://doi.org/10.15585/ mmwr.ss6612a1

5. Burger RJ, van Eijk AM, Bussink M, Hill J, Ter Kuile FO. Artemisininbased combination therapy versus quinine or other combinations for treatment of uncomplicated Plasmodium falciparum malaria in the second and third trimester of pregnancy: a systematic review and meta-analysis. Open Forum Infect Dis 2015;3:ofv170. https://doi.org/10.1093/ofid/ ofv 170

6. McGready R, Brockman A, Cho T, et al. Randomized comparison of mefloquine-artesunate versus quinine in the treatment of multidrugresistant falciparum malaria in pregnancy. Trans R Soc Trop Med Hyg 2000;94:689-93. https://doi.org/10.1016/S0035-9203(00)90235-9 
7. McGready R, Cho T, Keo NK, et al. Artemisinin antimalarials in pregnancy: a prospective treatment study of 539 episodes of multidrugresistant Plasmodium falciparum. Clin Infect Dis 2001;33:2009-16. https://doi.org/10.1086/324349

8. McGready R, Ashley EA, Moo E, et al. A randomized comparison of artesunate-atovaquone-proguanil versus quinine in treatment for uncomplicated falciparum malaria during pregnancy. J Infect Dis 2005;192:846-53. https://doi.org/10.1086/432551

9. Piola P, Nabasumba C, Turyakira E, et al. Efficacy and safety of artemether-lumefantrine compared with quinine in pregnant women with uncomplicated Plasmodium falciparum malaria: an open-label, randomised, non-inferiority trial. Lancet Infect Dis 2010;10:762-9. https://doi.org/10.1016/S1473-3099(10)70202-4

10. Kaye DK, Nshemerirwe R, Mutyaba TS, Ndeezi G. A randomized clinical trial comparing safety, clinical and parasitological response to artemether-lumefantrine and chlorproguanil-dapsone in treatment of uncomplicated malaria in pregnancy in Mulago hospital, Uganda. J Infect Dev Ctries 2008;2:135-9. https://doi.org/10.3855/T2.2.135

11. Mosha D, Guidi M, Mwingira F, et al. Population pharmacokinetics and clinical response for artemether-lumefantrine in pregnant and nonpregnant women with uncomplicated Plasmodium falciparum malaria in Tanzania. Antimicrob Agents Chemother 2014;58:4583-92. https:// doi.org/10.1128/AAC.02595-14

12. Nyunt MM, Nguyen VK, Kajubi R, et al. Artemether-lumefantrine pharmacokinetics and clinical response are minimally altered in pregnant Ugandan women treated for uncomplicated falciparum malaria. Antimicrob Agents Chemother 2016;60:1274-82. https://doi. org/10.1128/AAC.01605-15

13. McGready R, Stepniewska K, Lindegardh N, et al. The pharmacokinetics of artemether and lumefantrine in pregnant women with uncomplicated falciparum malaria. Eur J Clin Pharmacol 2006;62:1021-31. https:// doi.org/10.1007/s00228-006-0199-7

14. Tarning J, McGready R, Lindegardh N, et al. Population pharmacokinetics of lumefantrine in pregnant women treated with artemether-lumefantrine for uncomplicated Plasmodium falciparum malaria. Antimicrob Agents Chemother 2009;53:3837-46. https://doi.org/10.1128/AAC.00195-09

15. Price RN, Uhlemann A-C, van Vugt M, et al. Molecular and pharmacological determinants of the therapeutic response to artemetherlumefantrine in multidrug-resistant Plasmodium falciparum malaria. Clin Infect Dis 2006;42:1570-7. https://doi.org/10.1086/503423
16. PREGACT Study Group. Four artemisinin-based treatments in African pregnant women with malaria. N Engl J Med 2016;374:913-27. https:// doi.org/10.1056/NEJMoa1508606

17. Ukah M, Badejoko O, Ogunniyi S, Loto O, Aboderin O, Fatusi A. A randomized trial of artesunate-amodiaquine versus artemetherlumefantrine for the treatment of acute uncomplicated malaria in pregnancy. Int J Gynaecol Obstet 2015;131:41-4. https://doi. org/10.1016/j.ijgo.2015.05.009

18. McGready R, Tan SO, Ashley EA, et al. A randomised controlled trial of artemether-lumefantrine versus artesunate for uncomplicated Plasmodium falciparum treatment in pregnancy. PLoS Med 2008;5:e253.

19. Sowunmi A, Oduola AMJ, Ogundahunsi OAT, et al. Randomised trial of artemether versus artemether and mefloquine for the treatment of chloroquine/sufadoxine-pyrimethamine-resistant falciparum malaria during pregnancy. J Obstet Gynaecol 1998;18:322-7. https://doi. org/10.1080/01443619867038

20. Manyando C, Mkandawire R, Puma L, et al. Safety of artemetherlumefantrine in pregnant women with malaria: results of a prospective cohort study in Zambia. Malar J 2010;9:249. https://doi. org/10.1186/1475-2875-9-249

21. Kovacs SD, van Eijk AM, Sevene E, et al. The safety of artemisinin derivatives for the treatment of malaria in the 2 nd or 3rd trimester of pregnancy: a systematic review and meta-analysis. PLoS One 2016;11:e0164963.

22. Dellicour S, Sevene E, McGready R, et al. First-trimester artemisinin derivatives and quinine treatments and the risk of adverse pregnancy outcomes in Africa and Asia: a meta-analysis of observational studies. PLoS Med 2017;14:e1002290.

23. Askling HH, Bruneel F, Burchard G, et al.; European Society for Clinical Microbiology and Infectious Diseases Study Group on Clinical Parasitology. Management of imported malaria in Europe. Malar J 2012;11:328. https://doi.org/10.1186/1475-2875-11-328

24. Lalloo DG, Shingadia D, Bell DJ, Beeching NJ, Whitty CJM, Chiodini PL; PHE Advisory Committee on Malaria Prevention in UK Travellers. UK malaria treatment guidelines 2016. J Infect 2016;72:63549. https://doi.org/10.1016/j.jinf.2016.02.001 